\title{
Impact des vagues sur les structures en encorbellement de faible altitude au-dessus de la mer
}

\author{
Alexandre CINELLO ${ }^{1}$, Vincent LAFON ${ }^{1}$, \\ Benjamin BAILLY ${ }^{1}$, Camille LAURAS ${ }^{2}$
}

\section{Océanide}

Port de Brégaillon - BP 63, 83502 La Seyne sur Mer, France

contact@oceanide.net; bbailly@oceanide.net

2. Schlaich Bergermann Partner

25 rue du général Foy, 75008 Paris, France

c.lauras@sbp.de

\section{Résumé :}

Dietmar Feichtinger Architectes (DFA) avec Buzzo Spinelli Architecture ont proposé pour réaliser un cheminement piéton contournant la citadelle de Bastia une structure en encorbellement à une faible altimétrie par rapport au niveau d'eau. Ce type d'ouvrage en milieu exposé est rare du fait de l'importance des efforts qui s'appliquent notamment en sous-face de la structure et de la technicité du génie civil à développer.

Ce cheminement, en cours de construction aujourd'hui, a requis en phase de conception la mise en œuvre de modélisations physiques 2D et 3D spécifiques et innovantes pour représenter fidèlement le relief complexe de l'éperon rocheux localisé à la base du rempart de la citadelle et pour mesurer les efforts hydrodynamiques sur la structure.

Le présent papier décrit la campagne d'essai 3D, les traitements et analyses spécifiques des mesures d'efforts qui ont été réalisées et l'exploitation des résultats pour le dimensionnement des parties de l'ouvrage en console.

\section{Mots-clés :}

Modélisation physique, Bastia, Encorbellement, Console, Efforts, Impact de la houle, Post-traitement des mesures, Ancrage

\section{Introduction}

OCEANIDE réalise régulièrement des études en modèle physique 3D pour mesurer les efforts et moments hydrodynamiques sur les structures en encorbellement. Ces données servent aux dimensionnements de la structure notamment leur ancrage et à optimiser les conditions d'opérations.

Les 3 derniers modèles réalisés sont illustrés en figure 1.

La présente publication concerne la modélisation de la $3^{\text {ème }}$ référence et l'exploitation qui en a été faite dans le cadre du projet ci-dessous mentionné. 


\section{Thème 4 - Ouvrages portuaires, offshore et de plaisance}

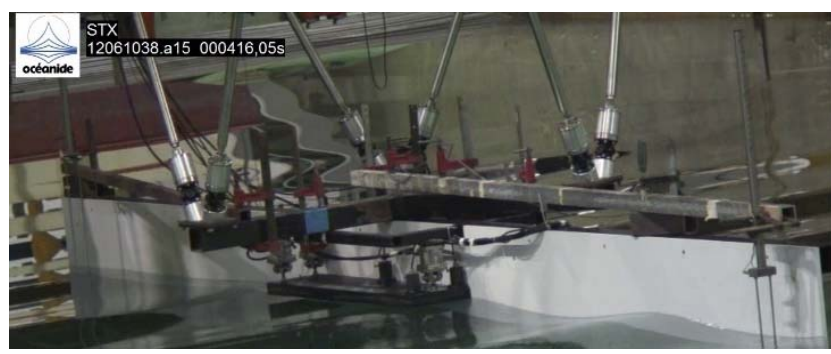

a) Plateforme mobile le long d'un navire.

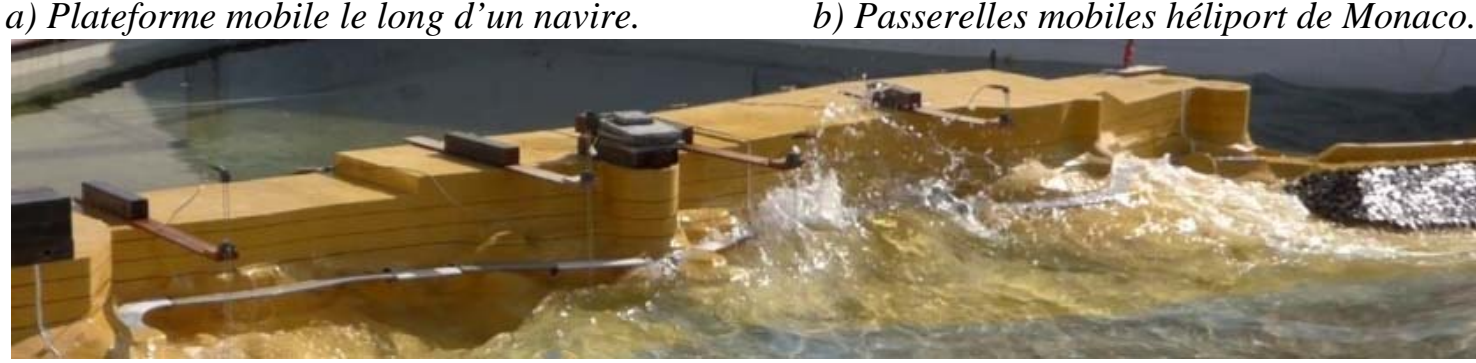

c) Cheminement piéton contournant la Citadelle de Bastia nommée l'Aldilonda.

Figure 1. Modèles 3D de structures en encorbellement (OCEANIDE).

Dietmar Feichtinger Architectes (DFA) est titulaire du marché de maitrise d'œuvre pour la réalisation d'un cheminement piéton de $480 \mathrm{ml}$ contournant la Citadelle de Bastia dont le rempart de protection contre la mer a été construit sur un éperon rocheux. DFA est associé à Buzzo Spinelli Architecture, au bureau d'étude structure Schlaich Bergermann Partner (SBP) et à OCEANIDE comme ingénieur hydrodynamicien.

La figure 2 présente les zones en encorbellements (en gris) et les zones creusées dans le rocher (en bleu) du cheminement en contre-bas du rempart de la Citadelle.

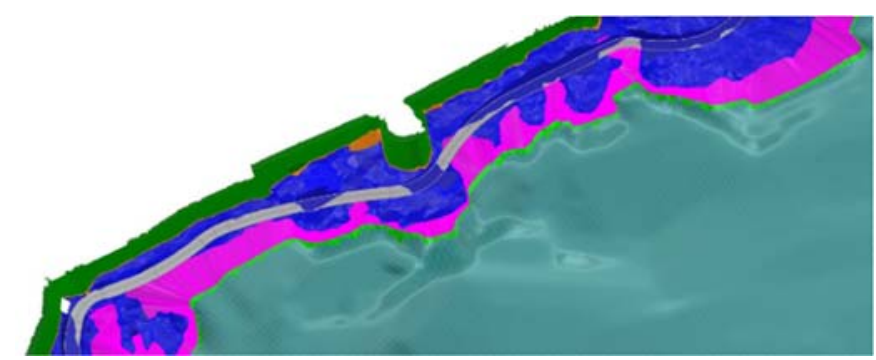

Figure 2. Implantation du cheminement en contre-bas du rempart de la Citadelle.

Sur la base de ce tracé (voir figure 2), les points critiques suivants ont été identifiés :

a) 4 sections en porte-à-faux où les impacts en sous-face de la structure en font des zones critiques en termes de dimensionnement;

b) 2 zones où le volume d'eau submergeant localement les zones creusées dans la roche conditionne le projet en termes de sécurité.

$\mathrm{Du}$ fait de la complexité des reliefs et des phénomènes physiques, les formules analytiques ou les modélisations mathématiques ne permettent pas de quantifier correctement les effets des vagues sur la structure. Pour ce qui concerne les aspects 


\section{XVİ̀mes Journées Nationales Génie Côtier - Génie Civil \\ Le Havre 2020}

hydrodynamiques, l'AVP de l'étude a consisté en une campagne d'essais 2D et le PROjet à une campagne 3D. Cette campagne 3D et l'utilisation qui en a été faite sont décrites ciaprès.

\section{Description des essais 3D}

\subsection{Modèle 3D}

La campagne d'essai 3D a été réalisée dans la cuve à houle d'OCEANIDE de $27 \mathrm{~m}$ de long et $12 \mathrm{~m}$ de large. Elle est équipée d'un batteur permettant de générer des houles de périodes de $0.5 \mathrm{~s}$ à $3 \mathrm{~s}$ et de hauteurs jusqu'à $0.6 \mathrm{~m}$ à 1 'échelle modèle.

Le modèle a été construit au $1 / 40^{\mathrm{ème}}$ et implanté pour une direction de houle incidente $\mathrm{N} 80^{\circ}$. L'ensemble du cheminement ainsi que l'enracinement de la jetée du Dragon au Nord et l'extrémité du rocher côté Sud ont été modélisés afin d'être parfaitement représentatif des conditions limites hydrauliques au droit du cheminement sur un linéaire de $325 \mathrm{~m}$.

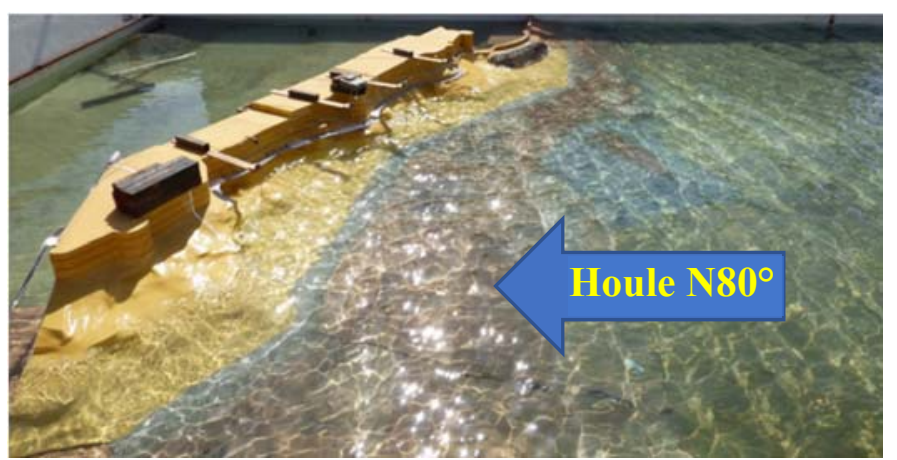

Figure 3. Implantation du modèle dans la cuve à houle.

La roche et le rempart, de la côte supérieure du rempart jusqu'à l'isobathe -10m NGF, ont été réalisés en blocs de polystyrène, par usinage à commande numérique, en intégrant les excavations dans le rocher pour le passage du cheminement et les réservations nécessaires à l'instrumentation de mesure (voir figure 3 - en jaune). La bathymétrie a ensuite été construite en contre-plaqué pour reproduire les fonds jusqu'à -25m NGF.

Le cheminement a été décomposé en plusieurs parties distinctes :

a) Les sections fixes (et non instrumentées) en encorbellement ou creusées dans le rocher ont été construites en bois et peintes en gris ;

b) Les sections instrumentées pour la mesure des efforts verticaux $\mathrm{Fz}$ et des franchissements (voir figure 4). 


\section{Thème 4 - Ouvrages portuaires, offshore et de plaisance}
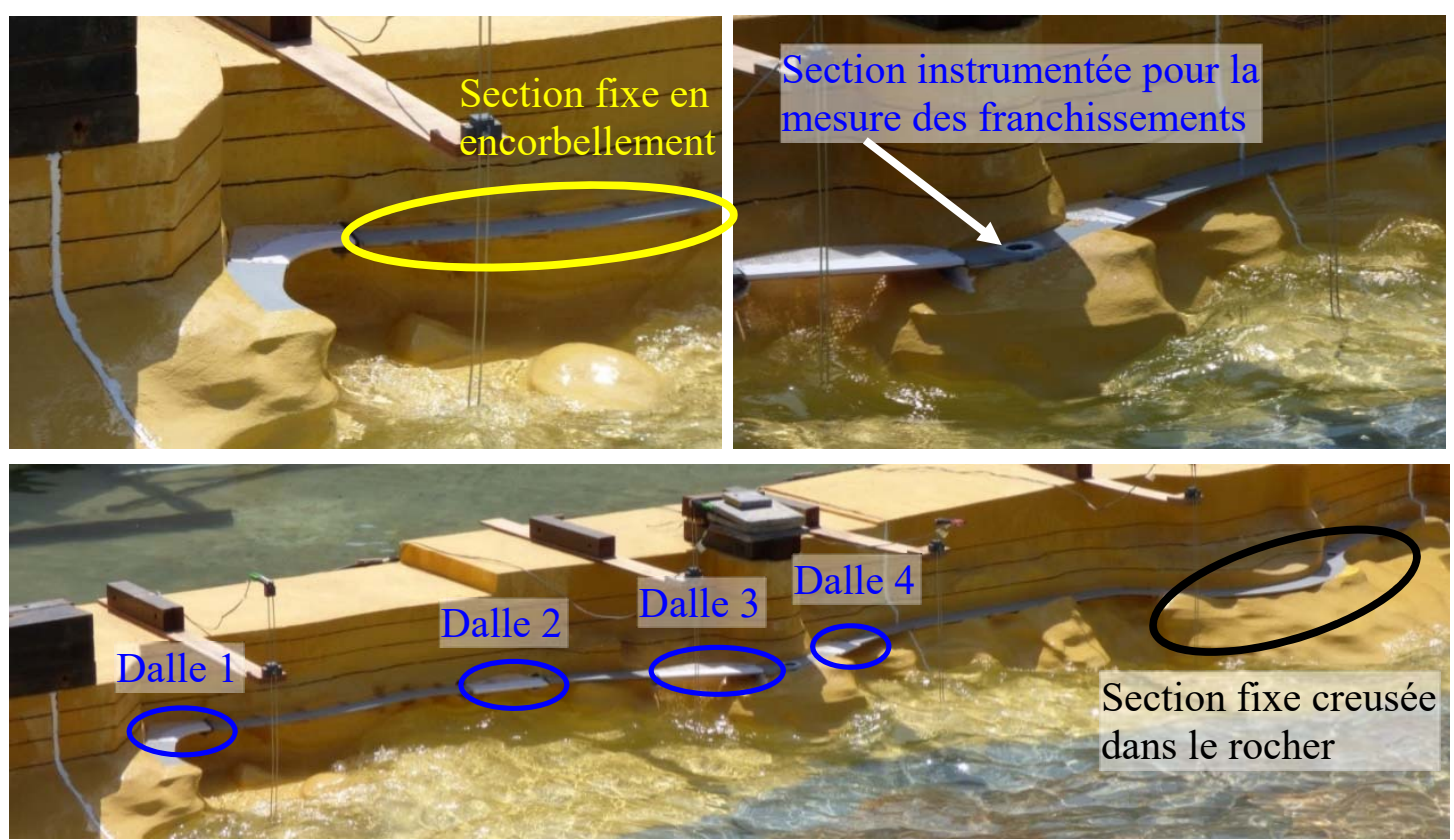

Figure 4. Sections fixes et instrumentées du cheminement modélisé.

Concernant les parties instrumentées en encorbellement (dalles 1 à 4), le tablier «principal» a été réalisé en PVC expansé blanc, le caillebotis a été modélisé par une plaque de polypropylène d'une porosité de $50 \%$ et les $1 / 2$ consoles positionnées en extrémité de chacune des sections instrumentées ont été réalisées en PVC. Ces dernières font parties intégrantes du cadre sur lequel le capteur d'effort est positionné. Le capteur étant lui-même monté sur un châssis acier fixé via une platine sur la dalle en béton du bassin.

2 configurations ont été étudiées pour les dalles, la figure 5 montre pour la dalle 1 la configuration initiale (avec caillebotis découpé selon le contour de la roche) et la configuration optimisée avec caillebotis de largeur constante.
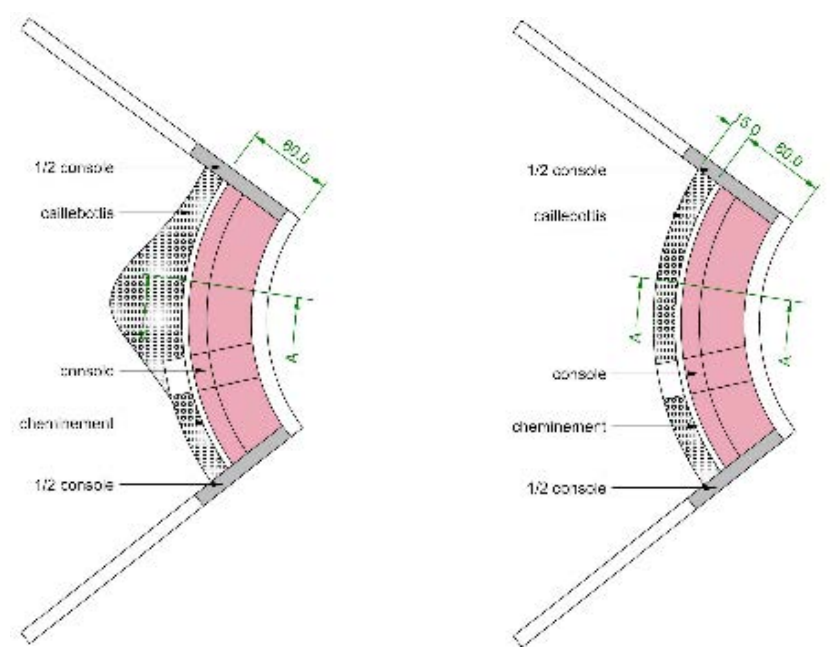

Figure 5. Détail de la Dalle 1 en configuration initiale et optimisée. 


\section{XVIèmes Journées Nationales Génie Côtier - Génie Civil \\ Le Havre 2020}

\subsection{Programme de houle}

Le programme de houle pour les 2 configurations est détaillé dans tableau 1 ci-dessous.

Tableau 1. Programme de houle.

\begin{tabular}{|c|c|c|c|c|c|c|c|c|}
\hline \multirow[b]{2}{*}{ Test $\mathbf{N}^{\circ}$} & \multirow[b]{2}{*}{$\begin{array}{l}\text { Configuration } \\
\text { testée }\end{array}$} & \multirow[b]{2}{*}{$\begin{array}{c}\text { Occurrence de } \\
\text { houle }\end{array}$} & \multicolumn{6}{|c|}{ Houle incidente - Specifiée } \\
\hline & & & $\begin{array}{c}\text { Houle } \\
{[-]}\end{array}$ & \begin{tabular}{|c|} 
Spectre \\
Jonswap
\end{tabular} & $\begin{array}{l}\mathrm{Hs} \\
{[\mathrm{m}]}\end{array}$ & $\begin{array}{l}\mathrm{Tp} \\
{[\mathrm{s}]}\end{array}$ & \begin{tabular}{|c|} 
Niv.Eau \\
[m NGF] \\
\end{tabular} & $\begin{array}{c}\text { Durée } \\
{[\mathrm{h}]}\end{array}$ \\
\hline 1 & Initiale & Operationnel 1 & Irréguliere & $y=3.3$ & 1.75 & 7.0 & 0.315 & 3 \\
\hline 2 & Initiale & Operationnel 2 & Irréguliere & $Y=3.3$ & 2.25 & 7.5 & 0.315 & 3 \\
\hline 3 & Initiale & Operationnel 3 & Irréguliere & $y=3.3$ & 2.25 & 10.0 & 0.315 & 3 \\
\hline 4 & Initiale & Operationnel 4 & Irréguliere & $Y=3.3$ & 2.50 & 8.0 & 0.315 & 3 \\
\hline 5 & Initiale/Optimisée & 1 an & Irréguliere & $\mathrm{V}=3.3$ & 3.00 & 8.0 & 0.515 & 3 \\
\hline 6 & Initiale/Optimisée & 10 ans & Irréguliere & $\gamma=3.3$ & 4.00 & 9.0 & 0.815 & 3 \\
\hline 7 & Initiale/Optimisée & 50 ans & Irréguliere & $y=3.3$ & 5.40 & 10.0 & 1.015 & 3 \\
\hline 8 & Initiale/Optimisée & 100 ans & Irréguliere & $y=3.3$ & 6.00 & 11.0 & 1.215 & 3 \\
\hline
\end{tabular}

\subsection{Mesures}

Les élévations de la surface libre ont été mesurées à l'aide de sondes à houles résistives. Un jeu de 5 sondes ( 1 à S5) a été positionné à 350 mètres au large du modèle par des fonds de -25 m NGF pour déterminer la houle incidente de chaque état de mer modélisé. L'élévation de la surface libre devant le cheminement et la falaise a été mesurée à l'aide de 4 sondes résistives (S6 à S9).

La mesure de l'effort $\mathrm{Fz}$ au niveau des 4 sections instrumentées a été réalisée à l'aide 4 capteurs d'effort 1D (1 par section), voir figure 6 .

Ces capteurs étanches permettent la mesure d'un effort dans une unique direction. La convention appliquée à la présente étude a été la suivante : un effort positif traduit un effort appliqué par les vagues sur le cheminement vers le haut.

Les 4 capteurs d'efforts ont permis la mesure des efforts en sous-face des sections instrumentées mais aussi des efforts sur la face supérieure du cheminement notamment induits par les retombées d'eau et/ou l'accumulations d'eau lors des franchissements.

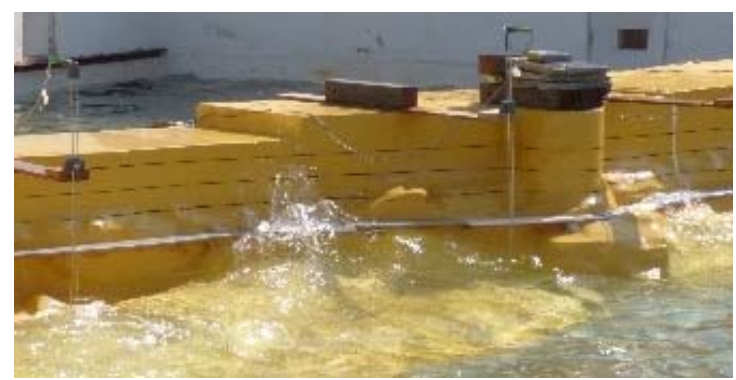

Figure 6. Illustration des surverses mesurées sur dalle 2 par les capteurs d'efforts Fz.

Chacun des capteurs d'effort 1D a été calibré puis positionné, monté et réglé verticalement par rapport au cheminement, i.e tablier principal et caillebottis, afin de ne mesurer que les efforts verticaux impactant le cheminement selon l'axe $\mathrm{Z}$. La période 


\section{Thème 4 - Ouvrages portuaires, offshore et de plaisance}

propre du montage global (i.e. la raideur du montage illustré ci-après : platine + châssis + capteur + cadre de reprise + cheminement) a été mesurée pour garantir une période propre bien inférieure à la période de la houle, voir figure 7 .

Les sections instrumentées du cheminement ont également été chacune et individuellement réglées horizontalement à la bonne altimétrie.

Les sections instrumentées ont été montées avec un jeu inférieur à $3 \mathrm{~mm}$ à l'échelle modèle afin d'éviter tout contact avec les parties fixes de la maquette, i.e section fixe du cheminement, rempart, roches.

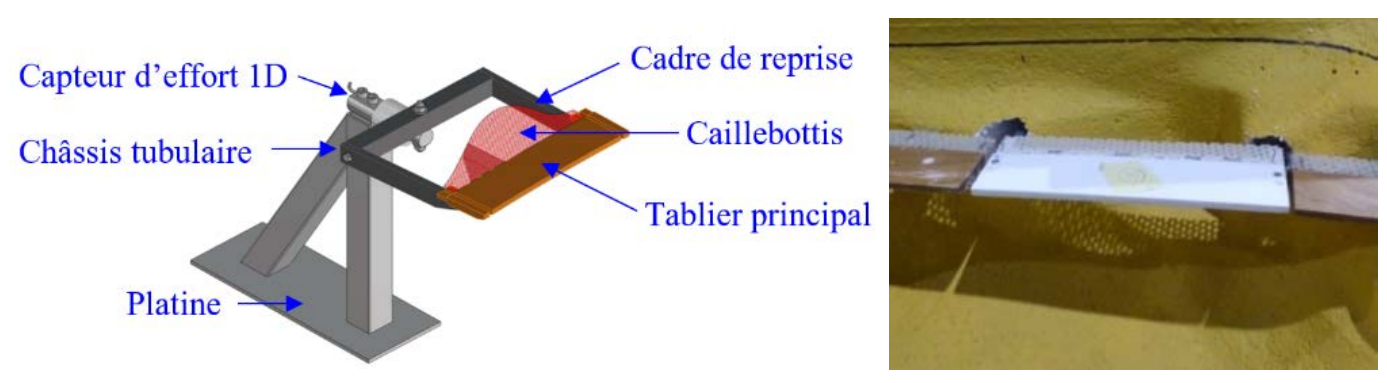

Figure 7. Instrumentation pour la mesure des efforts Fz.

Pour estimer la sécurité en haut de cheminement, les volumes de franchissement instantanés et moyens ont été mesurés en 2 zones en faisant communiquer une ouverture circulaire dans le cheminement avec un bac de réception situé derrière la maquette.

Afin de qualifier les hauteurs des impacts de la houle, des iso-lignes à $+7.5 \mathrm{~m},+10 \mathrm{~m}$ et +12.5 m NGF ont été représentées sur le modèle.

\section{Post-traitement des efforts}

La fréquence d'acquisition de la mesure des efforts est de $2000 \mathrm{~Hz}$ à l'échelle modèle. Un post-traitement spécifique est réalisé afin d'obtenir les efforts de prédimensionnement. En sus des efforts max mesurés lors de chaque essai, une analyse statistique des pics a été réalisée comme préconisé par le DNV-RP-C205 (2014). Ainsi, une loi statistique de Gumbel a été ajustée sur les valeurs extrêmes mesurées et les fractiles pour lesquels la probabilité d'occurrence est de $90 \%$ et de $95 \%$ ont été extraits.

Le tracé ci-dessous (voir figure 8) représente le signal de la voie traité (en rouge) et les pics extraits à partir d'une valeur seuil (cercles bleus). 


\section{XVI'̀mes Journées Nationales Génie Côtier - Génie Civil \\ Le Havre 2020}

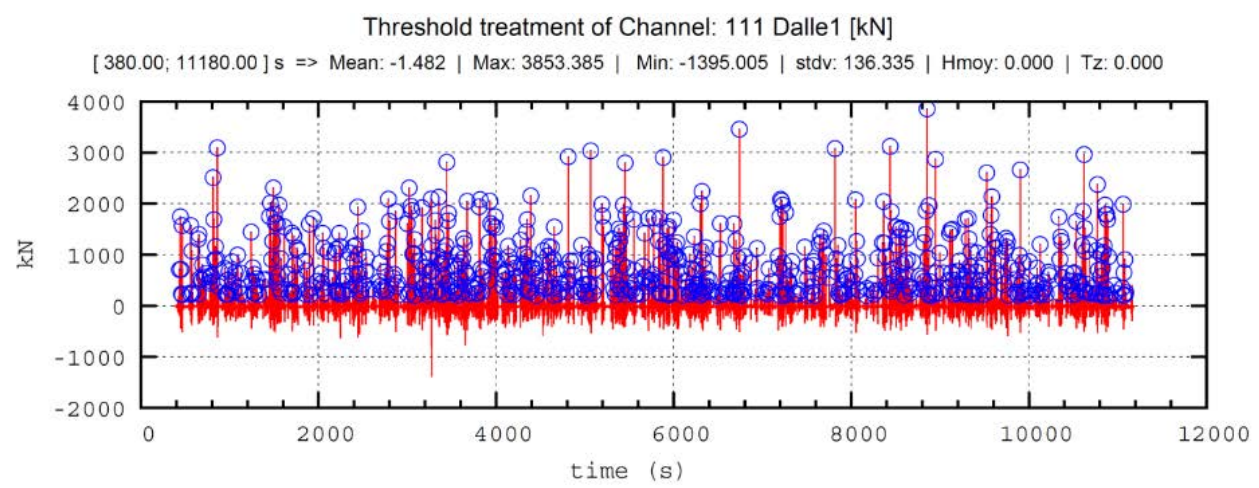

Figure 8. Sélection et extraction des pics à partir d'une valeur seuil.

Le second tracé ci-après (voir figure 9) compare la fonction de répartition des mesures (cercles bleus) à la fonction de densité cumulative pour la loi de Gumbel ajustée sur les valeurs extrêmes des résultats d'essais. Les résultats de cet ajustement sont rappelés en en-tête (Mean : Valeur moyenne des extrema, 90\%-val : Valeur pour laquelle $90 \%$ des extrema sont inférieurs, 95\%-val : Valeur pour laquelle 95\% des extrema sont inférieurs, A, B : Paramètre des lois de Gumbel).

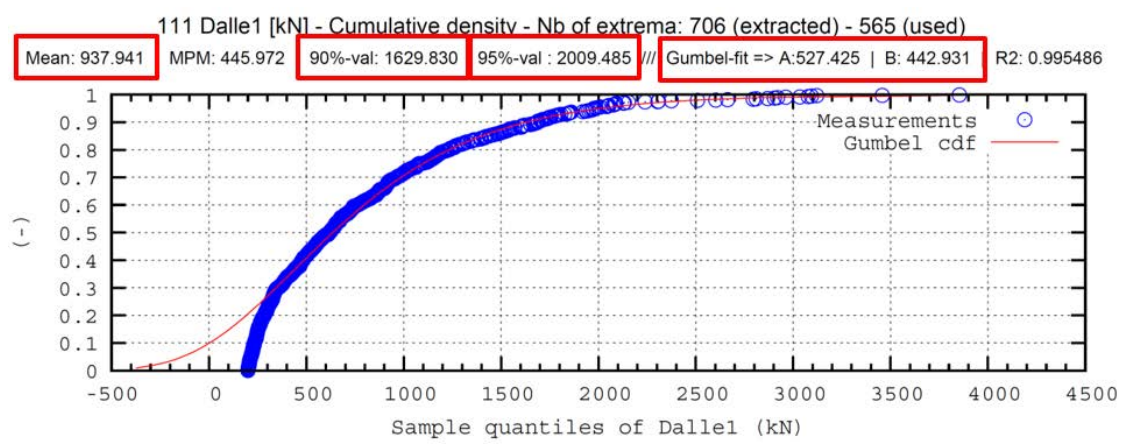

Figure 9. Calcul de la densité cumulative des valeurs de pic extraites.

\section{Observations des zones de focalisation de la houle}

En complément des mesures réalisées, des observations visuelles des impacts de la houle sur le cheminement ont été réalisées.
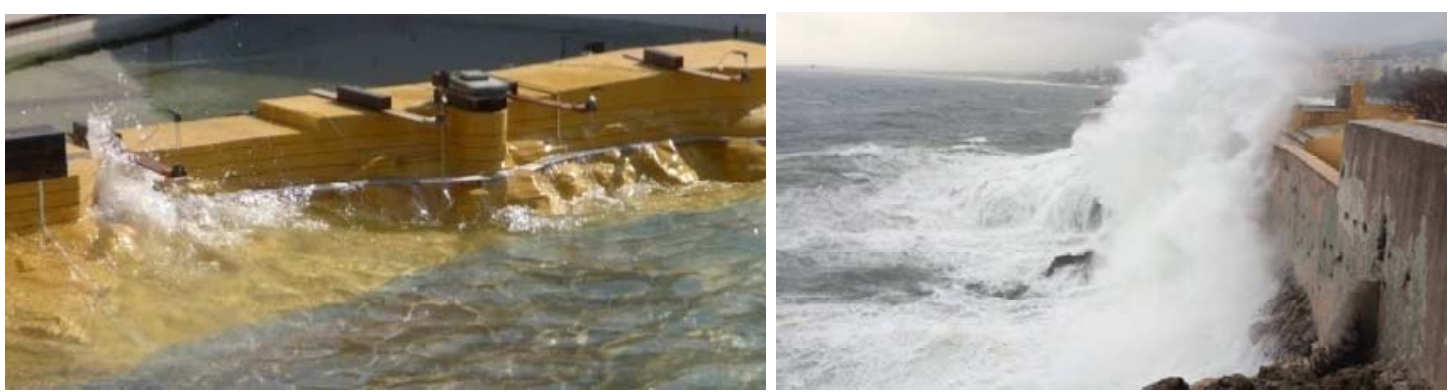

Figure 10. Observations visuelle impact - Houle 100 ans. 


\section{Thème 4 - Ouvrages portuaires, offshore et de plaisance}

La figure 10 illustre une des zones de concentration des vagues et montre une comparaison avec le phénomène existant en nature (tempête ayant eu lieu le 15 mars 2015).

\section{Exploitation des résultats d'essai}

Les résultats ont montré que la structure de l'ouvrage était destinée à reprendre des efforts verticaux ascendants et descendants particulièrement importants. Les efforts Fz obtenus pour les différentes dalles ont été convertis en charges par mètre linéaire de promenade, atteignant $600 \mathrm{kN} / \mathrm{ml}$ dans les zones de concentration de houle, soit une vingtaine de tonnes par mètre carré en cas de tempête centennale et environ $300 \mathrm{kN} / \mathrm{ml}$, soit une dizaine de tonnes par mètre carré en cas de tempête de période de retour de 50 ans.

Ces efforts ont été retenus comme hypothèses de charges dites accidentelles et caractéristiques, selon la terminologie des Eurocodes, tandis que les sollicitations considérées comme fréquentes ont été obtenues par interpolation linéaire pour correspondre aux charges de houle de période de retour de 20 ans.

La structure de l'ouvrage a été conçue et dimensionnée pour résister à ces charges et les faire transiter jusqu'à la roche et les remparts. Elle est composée d'une succession de consoles constituant la structure principale de l'ouvrage, ancrées directement dans la falaise ou dans le rempart, qui portent les tabliers avec lesquels elles forment, in fine, un ensemble monolithique en béton armé, figure 11. Les ancrages, au nombre de 5 par console ( 2 en partie haute, 3 en partie basse), sont précontraints.
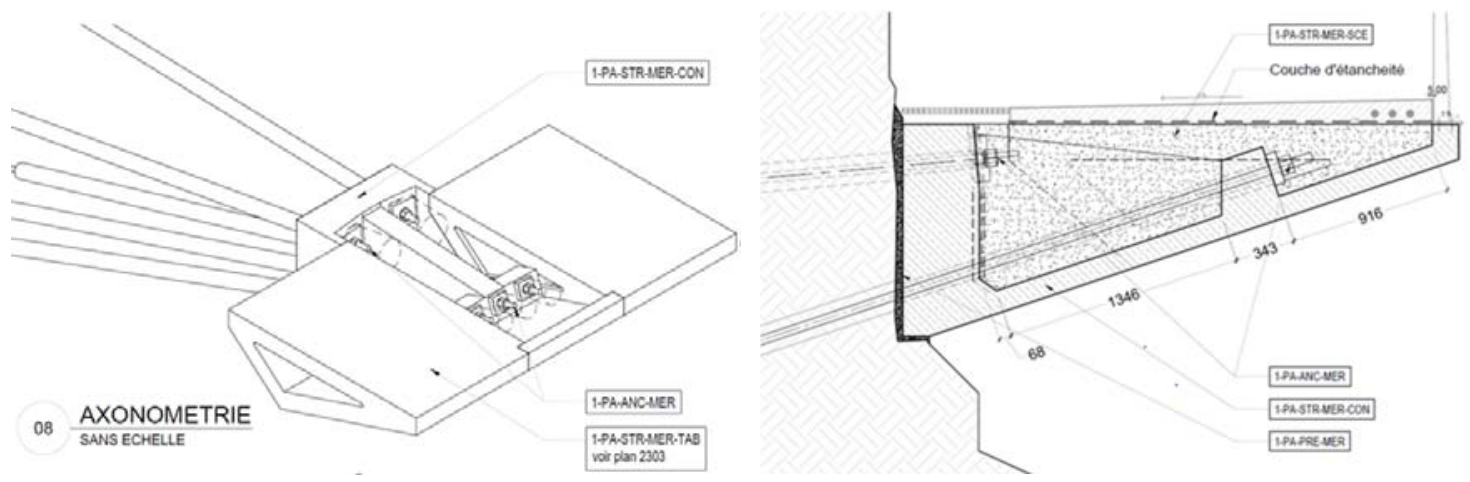

Figure 7. Vues de la console ancrée dans la falaise et tabliers.

Ainsi :

a) Les efforts verticaux ascendants induisent un moment de flexion dans les consoles, qui sollicite les ancrages inférieurs en traction et compriment la partie supérieure de la console ;

b) Les efforts verticaux descendants (dus aux retombées de vagues) induisent un moment de flexion qui sollicite les ancrages supérieurs en traction et compriment la partie inférieure de la console ; 


\section{XVIèmes Journées Nationales Génie Côtier - Génie Civil \\ Le Havre 2020}

c) Tous les efforts verticaux, ascendants et descendants, induisent un effort tranchant dans la console et du cisaillement à l'interface console/roche, repris par frottement entre la console et la roche, grâce à l'effet de compression induit par les ancrages.

En imposant un effort de compression permanent dans la console, la précontrainte des ancrages prévient à la fois le risque de fissuration excessive du béton et le décollement local de la console à l'interface avec la roche et protège ainsi les armatures et ancrages de la corrosion. Cette sollicitation permanente de la structure a été définie au cas par cas pour chacune des consoles, en fonction de l'exposition à la houle et de l'importance des retombées de celle-ci, grâce aux résultats des modélisations physiques en canal et bassin.

\section{Conclusion}

Tandis qu'un calcul analytique aurait permis de définir un effort conservatif à appliquer comme hypothèse de charge pour l'ensemble du cheminement, les modélisations physiques $2 \mathrm{D}$ et $3 \mathrm{D}$ ont permis de tester différentes configuration et conditions de projet. Ceci a été particulièrement utile pour la définition des efforts de précontrainte, puisqu'ils sont une sur-sollicitation permanente de la structure pour prévenir un risque, qui ne présente aucun avantage à être surestimée.

Au-delà d'être déterminants pour la forme de l'ouvrage, en démontrant par exemple l'intérêt d'évents sous forme de caillebottis le long de la promenade, de tels essais sont donc indispensables pour définir les hypothèses de charges auxquelles la structure de l'ouvrage doit résister.

\section{Références bibliographiques}

DNV-RP-C205 (2014). Environmental conditions and environmental loads, April 2014. 
Thème 4 - Ouvrages portuaires, offshore et de plaisance 